\title{
EL TRIPLE DESARROLLO DE SEGISMUNDO: ONTOGENIA, FILOGENIA Y DETERMINISMO SOCIAL
}

\author{
Alejandra Juno Rodríguez Villar \\ Duke University \\ Department of Romance Studies \\ 413 Chapel Drive \\ 205 Languages Building, Box 90257 \\ Durham, NC 27708 \\ USA \\ ar104@duke.edu
}

[Anuario calderoniano (ISSN: 1888-8046), 5, 2012, pp. 101-114]

La vida es sueño es una obra que se desarrolla en varios registros, por lo que no es recomendable limitar su lectura a su faceta teológica, moral o jurídico-política. En conjunto y de modo paralelo a los juegos de poder o a la disquisición sobre la decepción de los sentidos, esta obra dramática explora la evolución de un ser humano, de una persona y de un príncipe siguiendo un patrón antropogónico. $\mathrm{O}$ lo que es lo mismo, desde un animal a un hombre, desde un niño a un adulto y desde un prisionero a un príncipe. Siendo «evolución» la palabra clave, en este artículo utilizaré conceptos neurocientíficos para explicar este triple desarrollo. La neurociencia es la rama del saber que 
se ocupa del estudio del sistema nervioso y que en la actualidad, lejos de ser tan solo una rama de la biología, se postula como una ciencia interdisciplinar, con la que colaboran otras disciplinas como la lingüística, la filosofía o la psicología ${ }^{1}$. Por su parte, trabajos pioneros como el de Margaret R. Greer sobre las neuronas espejo y el código del honor en el Siglo de Oro español abren un nuevo campo de estudio al relacionar la materia literaria con la novísima disciplina neurocientífica ${ }^{2}$. La neurociencia estudia no solo cómo funciona el sistema nervioso del ser humano, sino también cómo se desarrolla ese mismo sistema e interacciona con la realidad circundante. Por ello, en este trabajo se tratará cómo La vida es sueño también puede ser entendida como el relato de esa triple evolución, convergiendo los tres aspectos en el hombre perfecto o acabado que Calderón nos sugiere como modelo social.

Ana María Enebral Casares sostiene, basándose en las estrechas relaciones entre el auto manuscrito, la comedia (1635) y el auto representado (1673), cómo la alegoría del camino recorrido por el hombre — creación, pecado, redención - es aún visible en La vida es sueño ${ }^{3}$, sin por ello menoscabar las lecturas posibles alejadas de la religión. Si la creación es seguida por el pecado original y con él aparece el sufrimiento del hombre, fuerza sería representar al protagonista en una torre de la que solo podría salir a través de la redención, que en este caso viene dada por el principio clásico de la victoria sobre las pasiones, el vencerse a uno mismo.

De esta manera, la narración cristiana no sería más que otro relato sobre la expulsión del paraíso y el camino de perfeccionamiento interior que cada ser humano recorre. Desde la caída en desgracia de Adán y Eva, pasando por la división platónica de las almas, hasta los mitos prometeicos, existe globalmente un eco de escisión del todo,

${ }^{1}$ Para estudiar las relaciones entre Neurociencia y Filosofia, ver Churchland, 1986; entre Neurociencia y Psicología, ver Kosslyn, 1994; y entre Neurociencia y Lingüística, ver Dehaene, 2009.

${ }^{2}$ En el caso concreto citado, Greer estudia el mecanismo de emoción pre-conceptual y pre-lingüística que el teatro provoca en el espectador, y que funciona también entre los personajes, y cómo ese mismo mecanismo hace asimismo del honor un tema muy exitoso en el teatro barroco. Véase el artículo de dicha investigadora contenido en este mismo número.

${ }^{3}$ Enebral Casares, 1993. 
que responde a la tesis esgrimida por el psicólogo Richard W. Coan cuando sostiene que muchos de los temas recurrentes en las diferentes mitologías pueden ser interpretados como estadios del desarrollo de la consciencia humana, y que ésta y la mitología puede que se hayan desarrollado de forma pareja en el tiempo ${ }^{4}$. Nos enfrentamos ahora con la evidencia de que, en numerosas ocasiones, el arte ha intuido certeramente circunstancias en las que solo tiempo después la ciencia ha recalado, permitiéndonos vislumbrar la concepción orgánica del arte que sostenía Aristóteles. El arte se revela así como continuo de las experiencias fisicas, como la expresión social de procesos internos que a veces no llegamos a aprehender conscientemente, como influencia en la vivencia física, explicable científicamente, y al mismo tiempo, como testigo poético de la misma.

Es conveniente recordar en estas páginas la obra del biólogo alemán Ernst Haeckel ${ }^{5}$, quien introdujo la idea de la «Ley Biogenética Fundamental» (sin ser en sí misma una ley, sino una hipótesis sin comprobar), según la cual a cada proceso ontogenético de cada ser humano corresponde un proceso filogenético de su especie ${ }^{6}$. La ontogenia se refiere al desarrollo del individuo desde el momento de su concepción hasta alcanzar la categoría adulta, mientras que la filogenia se refiere a todos los estadios por los que ha pasado la especie hasta llegar a su estado presente. Según Haeckel, cada individuo experimentaría y replicaría en su propio ser los cambios que su especie ha sufrido. Esta «Ley Biogenética Fundamental» está en la actualidad en desuso y, aunque sí que es cierto que el individuo pasa por diferentes fases, no se puede encontrar un paralelismo pleno y completo con la historia evolutiva de la especie. Sin embargo, este relato entrelazado entre la ontogénesis y la filogénesis se ha constituido como una potente metáfora reforzada por la deriva evolucionista en la que nos encontramos instalados desde la aparición de las tesis darwinianas.

La fallida hipótesis de Haeckel no pasaría de ser una mera metáfora más si no fuera por la inclusión reciente en nuestro vocabulario,

4 Coan, 1987.

${ }^{5}$ Ernst Haeckel (1834-1919) fue un gran seguidor de Darwin y el principal introductor de sus ideas en España, llegando su figura casi a eclipsar a la del naturalista británico en nuestro país.

${ }^{6}$ Para una más amplia información, ver Gould, 1977. 
de la mano de disciplinas como la neurociencia, de términos como el "cerebro reptil», el «sistema límbico» o el «neocórtex», nombres que se les da a distintas partes del cerebro y que, según nos asegura la ciencia contemporánea, son responsables de diferentes funciones vitales. En su obra The triune brain in evolution: role in paleocerebral function, el neurólogo Paul MacLean propone un modelo cerebral basado en la diferenciación de las partes del cerebro no solo por zonas, sino asimismo por grados de evolución o aparición ${ }^{7}$. Cada una de estas zonas habría sido superada y recubierta por otra posterior, encargada de funciones más complejas. Este cerebro triuno estaría compuesto en primer lugar por la zona más arcaica, llamada cerebro reptil y presente también en formas de vida consideradas inferiores» $^{8}$. El cerebro reptil o paleomamífero se ocuparía, según este modelo, de los instintos y de los resortes básicos para asegurar la vida. Sus conductas resultarían de difícil modificación porque están «pre-programadas» y son inconscientes y automáticas. Este cerebro rige la preservación de la especie y los cambios fisiológicos necesarios para la supervivencia. El cerebro reptil no piensa ni siente, sino que es puro instinto: responde con el resorte mecánico de la aceptación o el rechazo y se caracteriza por la acción.

De más reciente aparición es el sistema límbico, responsable sobre todo de las emociones, de la alegría, la tristeza, el dolor, el placer, el sexo, los lazos sociales o la empatía. Este sistema aparece en los mamíferos y trabaja en sintonía con el cerebro reptil: siente y desea. También es posible que la percepción del yo se forme en el sistema límbico, exactamente en el hipocampo, donde también se encuentra la memoria, fundamental en el proceso de auto-percepción.

Finalmente, el área más moderna en nuestro cerebro es el citado neocórtex. Esta parte del cerebro es característica del ser humano y de animales muy evolucionados. Esta parte es la que "piensa», resuelve problemas e imagina, y es donde se da el lenguaje y la creatividad.

7 MacLean, 1990.

${ }^{8}$ Hay que señalar al respecto que la moderna zoología está poniendo en tela de juicio las tradicionales divisiones entre seres vivos al descubrir formas sociales complejas en especies donde no parecía posible, por lo que hemos de ser cautos en este tema y reconocer que una de las grandes preguntas de este siglo será precisamente «¿qué es un ser humano?». Hoy se tambalea la clásica doctrina aristotélica según la cual lo que distingue al hombre de los otros animales es el ejercicio de la razón. 
Hace el ejercicio de anticipar el devenir a través de la información que recaba el cerebro de las funciones cognoscitivas. También es el responsable del pensamiento analítico y del operador binario, en términos de Eugene D'Aquili ${ }^{9}$. Esto quiere decir que el neocórtex divide el mundo en contrarios, lo que nos ayuda a interpretarlo.

A medida que nos desplazamos desde las zonas más arcaicas a las más nuevas del cerebro, más se va perdiendo la visión holística, global de la vida, y más se va configurando la idea del yo como individualidad alejada del todo. Este yo se caracteriza por estar enfrentado al mundo y a la realidad, por la desconexión del exterior y por el despertar de la conciencia individual. Esto explicaría por qué las religiones, por término general, hablan de esa escisión con el todo y su objetivo fundamental es volver a esa sensación de unión con el todo: el camino que va desde la expulsión del paraíso a la reunión de nuevo con el Padre. De ahí que, cuanto más analítico sea el pensamiento, menos tendencia se observa al pensamiento mágico o mítico. En las primeras fases, el ser humano es parte de la naturaleza; después se produce la separación y la naturaleza pasa a ser un objeto del que nos hallamos alienados y sobre el que no nos queda más remedio que reflexionar. En términos históricos y metafóricos, referidos al momento que le tocó vivir a Calderón, bien podríamos decir, y siempre dentro del relato cristiano, que el paso de la Edad Media a la Primera Modernidad marca ese paso del pensamiento holístico al analítico. El ser humano deja de sentirse parte del todo, donde el conocimiento no presenta fisuras, pues el libro de la naturaleza está escrito por el dedo de Dios, y pasa a la inseguridad que la quiebra de la palabra sagrada supone, dejando a los hombres solos ante su finitud y ante su absoluta ignorancia, constatando la diferencia que hay entre el yo y el resto. Para Calderón y sus contemporáneos no queda más opción que acercarse a la Naturaleza como a un objeto e intentar descifrar sus misterios, con la sensación de que ya nunca podrán volver a ella. Este esquema correspondería asimismo a la propia composición de La vida es sueño, donde Calderón ha compuesto un argumento jugando en todo momento con la oposición de contrarios, cuyo ejemplo más paradigmático es el de la oposición compuesta de hombre y fiera (dos

9 D'Aquili y Newberg, 1999. D'Aquili es un pionero del estructuralismo biogenético, las bases neurofisiológicas de la conducta humana. 
esencias distintas, contradictorias), en la que una acabará dominando a la otra.

Sin embargo, este relato neurocientífico también puede ser encontrado en otros registros. Informativa al respecto es la obra del lingüista Jean Gebser Ursprung und Gegenwart ${ }^{10}$, donde el autor propone que la conciencia ha pasado por diferentes estadios: arcaico, mágico, mítico y mental-racional, mutaciones evolutivas que se habrían dado por interacción con el entorno, como le sucede a Segismundo. Para este autor, la primera fase se caracterizaría por un estado previo a la consciencia; la segunda fase, por la falta del yo, la falta del espacio y del tiempo, por un mundo pleno de sentido, unitario, la unión con la naturaleza, y la reacción mágica a esa unión; la fase mítica, por la aparición del símbolo y el inicio de la escisión del yo, y la fase mentalracional respondería al descubrimiento de la causalidad.

Tras hacer el ejercicio de unir todos estos modelos, podemos volver a la idea de que Calderón, a través de la alegoría religiosa, está haciendo en La vida es sueño una recapitulación ontogenética y filogenética del hombre. Y no solo eso, Calderón está marcando al mismo tiempo un camino por el que seguir en el modelo social. En otras palabras, Calderón está explicitando lo que él, en relación a toda una tradición de pensamiento occidental, considera que es el óptimo desarrollo del ser humano. Como veremos a continuación, el dramaturgo madrileño no nos propondrá un modelo emocional y límbico, sino que, muy al contrario, desea un futuro racional, analítico y alejado del sentimiento, que se asemeja en no poco a la Ilustración que aparecerá en el siglo XVIII y al positivismo del siglo XIX.

Desde ese punto de vista, como ha sugerido Juan Luis Suárez en su artículo sobre la complejidad informacional en La vida es sueño, esta obra se entiende desde el final y no desde el principio, dado que el autor quiere justificar un punto de llegada ${ }^{11}$. A lo que asistimos es al proceso evolutivo de un ser humano, un hombre y un príncipe. Todo lo que sucede está encaminado a alcanzar ese punto de madurez que funciona como modelo presente de existencia y que tiene mucho que ver con el abandono de la visión holística-emocional en favor de la analítica-racional.

${ }^{10}$ El título podría ser traducido como Origen y presente. Ver Kramer, 1992.

11 Suárez, 2004. 
En cuanto a la división cerebral sugerida por MacLean, cada parte del cerebro es de diferente aparición cronológica, pero todas funcionan conjuntamente, aunque algunas funciones arcaicas sean inhibidas por funciones más recientes. De la misma manera, el Segismundo de La vida es sueño mantendrá comportamientos de las tres especies durante todo el texto (el caudillaje del «bando» de los rebeldes que se alzan por la libertad de Segismundo en la parte final de la obra es un reflejo de la función del cerebro reptil; se trata de la supervivencia a través de la acción, vv. 2373-2382), porque de no ser así no solo no tendríamos un personaje redondo, sino que además Segismundo se convertiría en poco operativo para el propósito dramático de la comedia. Aún así, el autor pone especial hincapié en la evolución de unas características en relación con otras.

Segismundo es un compuesto de hombre y fiera, pero en realidad es un príncipe. Sabe quién es, pues hay en él una grandeza del alma innata por ser hijo del rey ${ }^{12}$. Aunque preso, ha sido educado en las artes liberales, recurso que Calderón utiliza para justificar esa potencialidad a un nivel dramático: «solo Clotaldo / le ha hablado, tratado y visto: / éste le ha enseñado ciencias» (vv. 754-756). Aunque en un primer nivel creamos que ambas facetas - la del hombre y la de la fiera- están compensadas, el autor juega desde el primer momento con el ente posible, es decir, un ser humano completo, un ente no existente pero con la aptitud para existir. Calderón juega con la potencia absoluta y natural: de ahí que la obra haya de ser entendida desde su final, como la historia del desarrollo / adquisición de las características humanas. Asistimos a la potencialidad que un niño tiene de ser un adulto, que un ser inferior tiene de convertirse en un ser humano y que un prisionero tiene de ser un príncipe (o un esclavo de ser un hombre libre, un hombre que puede elegir). En definitiva, asistimos como espectadores a una potencia ontogenética, filogenética y social, con coincidencia de las tres. $\mathrm{O}$, desde una perspectiva neurocientífica, es el paso desde el cerebro reptil al neocórtex, con la seguridad de que se llegará a ese ente actual. En el nivel estructural, Calderón pre-

12 Podemos ver en La vida es sueño una alusión a la scala naturae, que habla del orden jerárquico en el que Dios ha dividido la Naturaleza con una idea clara de progresión que a nivel terrenal culmina con el ser humano en su forma de rey, debajo del cual estaría el príncipe. Ver al respecto Lovejoy, 1974. 
senta la información de una forma dual, jugando con el binomio del acto y la potencia, solo posible en el desarrollo temporal, cuya ruptura por parte de Basilio constituirá el mayor obstáculo para el protagonista. Pero aún así, la presentación de las oposiciones cognoscitivas (realidad / sueño) dará lugar a un pensamiento binario, racional, capaz de separar el yo del entorno (del destino universal de las áreas más arcaicas del cerebro) y, por lo tanto, de dominar las reacciones instintivas. En otras palabras, de la acción y del sentir se pasará al pensar.

La trama, desde el punto de vista del desarrollo interior del personaje, nos presenta a un Segismundo muy consciente de su yo desde su primer parlamento (vv. 102-172). El «soy quien soy» está ligado a su cualidad de humano, pero aún no de adulto o príncipe. Aunque Rosaura se refiera a Segismundo como «nacido entre las fieras» (v. 1658), lo cierto es que Calderón nos presenta al protagonista cuando ya se ha producido la escisión desde el todo, de la conexión con el «ser», y la expulsión del paraíso. Pese a ello, hay en su discurso inicial un deseo de reencuentro con esa totalidad, que se expresa a través de la comparación con los seres inferiores, "a un pez, a un bruto y a un ave» (v. 172). Existe la continuidad en la Cadena del Ser y Segismundo se reconoce como un eslabón más de la misma, aunque carente de algo que, por estar por encima (pues tiene "más alma», «más albedrío» y «más vida» que los seres inferiores, vv. 131, 151 y 161), le corresponde más que al resto: la libertad. Hay una conexión directa del personaje con la Naturaleza, tal y como se da en las zonas más arcaicas del cerebro. Aunque hay reflexión en este primer monólogo, es sobre todo una reflexión sobre la emoción; Segismundo está sintiendo:

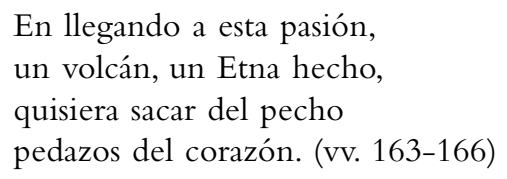

Aparece el personaje unido a su desdicha, sin posibilidad de actuación sobre la misma y formando parte de un destino más grande que él mismo, en un todo holístico escrito en las estrellas y cuyas primeras reacciones oscilarán entre emocionales y primarias. En este momento Segismundo amenaza ya con una muerte irracional, sin atender a razones, en un ejercicio de supervivencia al detectar la presencia amenazante de Rosaura: «Pues la muerte te daré/ [...] / que sabes fla- 
quezas mías» (vv. 180-182). Sin embargo, Rosaura, además de despertar en Segismundo el poder educador del amor, también se ofrece a nuestro protagonista como un igual en sufrimiento y desdichas. Comienza, pues, un proceso de empatía propio del sistema límbico, que ayudará a Segismundo a avanzar en su evolución pues aprende a establecer lazos emocionales con otros individuos. Rosaura certificará ese nexo entre los dos con la historia del sabio y su aplicación al encuentro con Segismundo:

\author{
Quejoso de la fortuna \\ yo en este mundo vivía, \\ y cuando entre mí decía: \\ ¿habrá otra persona alguna \\ de suerte más importuna? \\ piadoso me has respondido, \\ pues volviendo en mi sentido \\ hallo que las penas mías \\ para hacerlas tú alegrías \\ las hubieras recogido. (vv. 263-272)
}

A tal grado llega esta vinculación que Segismundo amenazará con «despedazarse» a fin de defender la vida de los nuevos personajes ante la llegada de Clotaldo solo instantes después (vv. 309-318). La empatía límbica se presenta así como el paso previo para la racionalidad neocortical que desarrollará Segismundo a partir de esa mencionada primera escena.

A la llegada al palacio, Segismundo reaccionará de manera emocional, empatizando de manera positiva o negativa y más que centrado en las reacciones instintivas de supervivencia (las propias del cerebro reptil), basándose en aquellas que le causan placer o dolor, tal y como corresponde al funcionamiento del sistema límbico. Sus respuestas siguen siguiendo inmediatas e irracionales: exige a los músicos que no canten más porque le desagrada su música de entretenimiento, se deleita con las adulaciones de Clarín y queda deslumbrado por la belleza de Estrella. Sin embargo, en la escena de palacio aún no ha habido una fractura del yo consciente, y el yo se necesita intacto para completar la evolución. Será tras la vuelta a la torre cuando todo este proceso se desencadene a través de la perturbación de la constitución del yo consciente, entidad que solo puede ser posible en el 
desarrollo temporal y espacial, atributos del neocórtex. Esa perturbación a la que Basilio le somete hurtándole unidades de información espacio-temporal con el uso de narcóticos crea en Segismundo la sensación de sueño ${ }^{13}$. Dado que en el sueño no existen esas categorías, sino que están suspendidas, se convierten en improcesables informacionalmente, pero al mismo tiempo el sueño nunca llega a existir realmente y por eso la experiencia siembra las incógnitas en el protagonista. Aun así, Calderón construye para el espectador la escena del palacio con la marca del sueño, pues presenta este pasaje falto de guía moral y de consecuencias (dentro del mismo plano del palacio). Segismundo aún no ha dado aquí el salto al neocórtex, pues todavía no maneja la relación causa-efecto. Para el espectador la escena se configurará como un sueño, con su amoralidad y su carga sexual. Para Segismundo será una experiencia real que activa una búsqueda que restaure el orden interno, tras la vuelta a su prisión.

La evolución de Segismundo, la evolución del individuo, la evolución del ser humano, es algo que depende de la interacción directa con el entorno, situación que le ha sido hurtada hasta la primera salida de la torre. La experiencia en el palacio, el proceso de simulación al que Basilio somete a Segismundo, no es tan definitiva a ese nivel como la ruptura del yo que provoca. Segismundo ha de reflexionar sobre los mecanismos de la realidad exterior, establecer relaciones de causalidad: se muestra así el desarrollo del neocórtex. Antes la realidad era percibida como inamovible y el nacimiento como castigo. Ahora, tras el paso aparentemente sonámbulo por palacio, esta misma realidad se exige transformable. En términos kantianos, hay una formación simultánea del yo y del mundo como objeto. Es después de la primera experiencia en el palacio cuando aparece más consciente el cómputo del tiempo, porque es después de esa experiencia cuando Segismundo ha de hacer su ejercicio de memoria para intentar establecer una continuidad del yo, que solo existe en la secuencia memorística y la memoria es una facultad solo realizable en una secuencia temporal. Segismundo se ve obligado desde ese momento a resolver

13 No olvidemos que, cuando Segismundo despierta de nuevo en la torre, sufre una desorientación espacial al no verse ya en palacio («Mas jay de mí! ¿̇ónde estoy?», v. 2077) y Clotaldo le hace pensar a continuación que «hora es ya de despertar» (v. 2091). 
los nuevos y complejos problemas que resultan de su capacidad cognitiva con el entorno, a completar los vacíos. Esto provoca la evolución, en cuyo estado primigenio podemos observar la manifestación de las funciones arcaicas del cerebro, como matar sin un sentido de justicia; quizás ese primer asesinato del criado no representa más que la resistencia a la escisión e individualización total del yo, a la consciencia racional (funcionando ese personaje como metáfora de la misma) a la que finalmente Segismundo se verá abocado. Al fin y al cabo, la concepción holística evita las consecuencias personalizadas, pues no existe la autonomía del yo consciente.

Como ya señaló E. M. Wilson ${ }^{14}$, será la información provista por Rosaura al reencontrarse con Segismundo tras su liberación de la torre (vv. 2690-2921), quien funciona como un dispositivo externo de la memoria ${ }^{15}$, con la que realmente se generará el proceso memorístico, necesario para la supervivencia de Segismundo como individuo, porque es la información que le ayuda a reconfigurar el yo consciente. La larga relación de Rosaura y su presencia misma frente a la torre es la información que colabora a la formación del sentido de la causalidad en Segismundo y, por lo tanto, de la percepción del sentido secuencial del tiempo, expresado a través de relaciones de causaefecto.

Es posible que Segismundo hubiera aceptado el relato de la protagonista femenina, aun no siendo cierto, en virtud de la poca tolerancia que el cerebro presenta ante las discontinuidades de la información ${ }^{16}$, por ser operativo para sus propósitos. En otras la palabras, ya no importa «la verdad». Le ayuda a ordenar temporalmente una información que estructura el yo y el personaje no puede actuar de otra manera. La «verdad» pasa de estar en el objeto al sujeto. Segismundo ya no buscará certezas, sino convicciones operativas: no se busca más la comunión con el todo, sino la convivencia con el entorno. Rosaura ayudará a Segismundo a conformar una conciencia autónoma, con una continuidad expresada en secuencias de espacio y tiempo que desembocan en la persona. Traerá la memoria semántica, la de los he-

14 Wilson, 1965.

15 En expresión de Merlin Donald (1991, p. 273).

${ }^{16}$ El cerebro completa la información incompleta artificialmente. El ejemplo más popular al respecto es el punto ciego del ojo. 
chos, que recompondrán la memoria episódica de Segismundo, la referida al yo en el tiempo, y por ello también llamada memoria autobiográfica, ambas capacidades albergadas en el sistema límbico. Es decir, se trata de un nuevo paso hasta la total evolución. Es el sistema límbico el que decide lo que se guarda en la memoria o no bajo criterios de emocionalidad y, por ello, el relato de Rosaura es válido para Segismundo. El protagonista construye su capacidad moral a partir de las consecuencias efectivas a nivel cognitivo de ese relato porque está unido a la emoción. Y superado ese punto, la ya existencia del módulo del «yo» escindido del entorno rige la sensación de control de nuestra vida mental. Sin el «yo» no hay libre albedrío y ahora Segismundo ha alcanzado el "yo» del individuo.

En fases posteriores se verán repeticiones de comportamientos, pero con la aplicación de un criterio analítico, de justicia comunal, sin embargo, alejado de la empatía límbica. Al principio Segismundo opera con un sistema holístico que, a fuer de no ser analítico y no expresarse en secuencias temporales, no puede prever las consecuencias. Tras su transformación, sus actos estarán encaminados a anticiparlas, lo que explicará el hecho de que Segismundo condene al soldado en una falta total de empatía pero en un ejercicio de previsión utilitaria para evitar posibles futuras rebeliones. Los sentidos no existen para reflejar la realidad sino para ayudar a la preservación individual y grupal: ésa es la lección de Segismundo, que aplica en el final de la obra, cuando se le reconoce como príncipe y soberano. No ha de utilizarlos para conseguir un retrato adecuado de la realidad, y más en este momento en el que, roto el libro de Dios, ya no puede existir tal cosa, sino para asegurar su supervivencia en sus tres facetas. El esquema perceptual social de Segismundo se basa al final de la obra en su ser como príncipe y con él completa los vacíos. Si su naturaleza en acto es la del hombre, su esencia es la de príncipe. En otras palabras, el Hombre aprehende el sentido moral y el Príncipe la ética que le hará sobrevivir. El operador binario del neocórtex se manifiesta aquí con la conciencia de que un príncipe y un soldado son dos «especies» diferentes. En ese momento de consciencia de la diferencia, Segismundo ha alcanzado su "yo» como príncipe. Paralelo al desarrollo natural se ha cumplido el determinismo social en el que, según el topos del Theatrum Mundi, cada uno ha de interpretar su papel adecuadamente. Desaparece así en Segismundo la empatía que, sin embargo, antes hemos visto con 
todo tipo de fieras. Esa comunión con el entorno cesa en virtud del enrocamiento del yo que deriva en un comportamiento racional-utilitarista, basado en la necesidad de tener amigos en cualquier circunstancia y perceptible en las diferentes decisiones que Segismundo toma con respecto a cada personaje principal, perdonando a Clotaldo y a su padre, condenando al soldado, casándose con Estrella y entregando a Rosaura a Astolfo. El objetivo del paleo-cerebro ha llegado a expresarse neocorticalmente. Es la reconstitución del «yo» del ser humano.

En conclusión, el sentido teleológico del tiempo en la cultura cristiana se solapa con la idea evolucionista, en la que los cambios se expresan en secuencias temporales progresivas, y ambos conceptos hallan eco en La vida es sueño. De una visión holística, conectado con la naturaleza y el destino, Segismundo pasa a una visión analítica en la que el medio ha de ser algo manipulable. De sobrevivir primariamente, aprende a empatizar y finalmente a razonar. Esa evolución, por omnipresente, se expresa en varios niveles, que en el caso de La vida es sueño responde a una alegoría social, ontogenética y filogenética, en la que el personaje principal se hace con los atributos que caracterizan al adulto, al ser humano y al príncipe. 


\section{BibLiOgRAFía}

Calderón de la Barca, P., La vida es sueño, ed. C. Morón Arroyo, Madrid, Cátedra, 2011.

Churchland, P., Neurophilosophy: Toward a Unified Science of the Mind-brain, Cambridge (Massachusetts), MIT Press, 1986.

Coan, R. W., Human Consciousness and its Evolution, New York, Greenwood Press, 1987.

D’Aquili, E. y Newberg, A., The Mystical Mind: Probing the Biology of Religious Experience, Minneapolis, Fortess Press, 1999.

Dehaene, S., Reading the Brain: The Science and Evolution of a Human Invention, New York, Penguin Viking, 2009.

Donald, M., Origins of the Modern Mind:Three Stages in the Evolution of Culture and Cognition, Cambridge (Massachusetts), Harvard University Press, 1991.

Enebral Casares, A. M., "Argumentos en pro de una reinterpretación de La vida es sueño», en Hacia Calderón. Décimo coloquio Anglogermano, ed. H. Flasche y K. Dirscherl, Stuttgart, Steiner, 1993, pp. 223-241.

Gould, S. J., Ontogeny and Phylogeny, Cambridge (Massachussets), Harvard University Press, 1977.

Kosslyn, S. M., Image and Brain: The Resolution of the Imagery Debate, Cambridge (Massachusetts), MIT Press, 1994.

Kramer, E. M., Consciousness and Culture: An Introduction to the Thought of Jean Gebser, New York, Greenwood Press, 1992.

Lovejoy, A. O., The Great Chain of Being: A Study of the History of an Idea, Cambrigde (Massachusetts), Harvard University Press, 1974.

MacLean, P. D., The Triune Brain in Evolution: Role in Paleocerebral Function, New York, Plenum Press, 1990.

SuÁrez, J. L. «El reconocimiento de la complejidad. Información dispersa y reconocimiento incompleto en La vida es sueño», Edad de Oro, 23, 2004, pp. 355-368.

Wilson, E. M, "On La vida es sueño», en Critical Essays on the Theater of Calderón, ed. B. W. Wardropper, New York, New York University Press, 1965, pp. 63-89. 